\title{
Reactivities of Non-protein Substances in a Modified Lowry Procedure Using Chloramine- $T^{\dagger}$
}

\author{
Masashi Higuchr and Fumio YoshidA \\ Laboratory of Biochemistry, Azabu Junior College of Public Health, \\ Sagamihara-shi, Kanagawa 229, Japan
}

Received January 25, 1978

\begin{abstract}
More detailed study on the reactivities of non-protein substances in a modified Lowry procedure using chloramine-T (CAT) has been performed. Many compounds, which hardly showed the color by the original procedure, became to react with the phenol reagent in the modification which used CAT in trichloroacetic acid (TCA) solution. However, the color yields obtained by this modification of certain of these compounds were markedly or considerably repressed when one of the sulfhydryl (SH) compounds coexisted in the reaction mixture. Moreover, the absorbances of phenols, tryptophan, tryptamine, several purine bases, and penicillin $\mathrm{G}$ which give the deep color by the original procedure were greatly or partially decreased by this modification.

On the other hand, in almost all the tested compounds, the absorbances obtained by the other modification which used CAT under the alkaline $\mathrm{pH}$ were not appreciably higher than those obtained by the original procedure.
\end{abstract}

Since Lowry et al. reported a micro method for protein determination with the use of the phenol reagent, ${ }^{11}$ this method has been one of the most widely used colorimetric protein measurement methods. This procedure, however, offers a disadvantage because of the high reactivities of the phenol reagent with many non-protein substances under the condition applied.

We have recently reported two simple modifications in Lowry procedure using CAT. One is the addition of CAT to the protein samples in the acid medium such as TCA before the addition of the alkaline copper reagent. This modification has an advantage in that the protein content can be measured in the presence of relative high levels of $\mathrm{SH}$ compounds or some other reducing agents. ${ }^{2}$ ) The other modification is the addition of CAT to the

\footnotetext{
+ Abbreviations for Good buffer: Bicine, N,N-bis(2hydroxyethyl)-glycine; BES, N,N-bis(2-hydroxyethyl)2-aminoethane; HEPES, N-2-hydroxyethyl(piperazine$\mathrm{N}$-2-ethanesulfonic acid; MOPS, 3-(N-morpholino)propanesulfonic acid; PIPES, piperazine- $N, N^{\prime}$-bis(2ethanesulfonic acid); CAPS, cyclohexylaminopropanesulfonic acid; Tricine, tris(hydroxymethyl)-methyl glycine; TES, N-tris(hydroxymethyl)methyl-2-aminoethanesulfonic acid.
}

protein samples after the addition of the alkaline copper reagent, which gives a more linear protein standard curve with a slight loss of sensitivity, compared with that given by the original procedure. ${ }^{3)}$ More detailed informations on the reactivities of non-protein substances in those modifications, however, yet remains to be presented. This paper presents the results of the experiment designed to obtain this information.

\section{MATERIALS AND METHODS}

Chemicals. L-Amino acids, and D-monosaccharides and their derivatives were used throughout all experiments. Amino acids were kindly gifted from Ajinomoto Co., Inc., Tokyo. DNA, RNA, glucose-1 and -6-phosphates, glucosamine, galactose, histamine, tryptamine, dithiothreitol, and crystalline bovine serum albumin(BSA) were obtained from Sigma Chemical Co., St. Louis, glucose was from E. Merck, Darmstadt, and penicillin $G(\mathrm{~K}$ salt) was from Calbiochem, La Jalla. Folin-Ciocalteu phenol reagent was a product of Wako Pure Chemical Co., Osaka, and was used after dilution with water to $1 \mathrm{~N}$ in acid. Other chemicals used were of the reagent grade.

Methods. Method I: This method was essentially the same as described previously. ${ }^{2)}$ To $0.5 \mathrm{ml}$ of aqueous solution containing BSA and/or one of the test 
compounds were added $0.25 \mathrm{ml}$ of $10 \%(\mathrm{w} / \mathrm{v}) \mathrm{TCA}$ and $4 \%$ CAT solutions. After the mixture was allowed to stand for $30 \mathrm{~min}$ at room temperature, $5 \mathrm{ml}$ of the alkaline copper reagent which contained $0.15 \mathrm{~N} \mathrm{NaOH}$ instead of $0.1 \mathrm{~N} \mathrm{NaOH}$ were added to the mixture. After $30 \mathrm{~min}$ at room temperature, $0.5 \mathrm{ml}$ of the phenol reagent were rapidly added to ensure mixing. Absorbance at $750 \mathrm{~nm}$ was read after $30 \mathrm{~min}$ at room temperature.

Method 1I: This method was essentially the same as described previously. ${ }^{3)}$ To $0.5 \mathrm{ml}$ of aqueous solution containing BSA and/or one of the tested compounds were added $0.25 \mathrm{ml}$ of water and $5 \mathrm{ml}$ of the alkaline copper reagent which was identical with that of the original method, then $0.25 \mathrm{ml}$ of $4 \% \mathrm{CAT}$ solution were added. This mixture was allowed to stand for $10 \mathrm{~min}$ at room temperature. Subsequent procedures were the same as those of Method I.

In both methods, a blank and a standard without CAT were run in parallel.

The amounts of the tested compounds shown in this paper were those in a sample volume of $0.5 \mathrm{ml}$.

\section{RESULTS}

\section{Results by Method I}

Amino acids and the related compounds. As shown in Table I, among the amino acids which give no color by the original procedure, arginine, aspartic acid, glutamic acid, lysine, methionine, phenylalanine, and valine became to react with the phenol reagent by Method I though the others gave very low absorbances. On the other hand, among the amino acids which show the blue color by the original procedure, asparagine and histidine gave greatly increased absorbances, and tryptophan and tyrosine showed a little lower absorbances. In general, the absorbances obtained by Method I of amino acids depended on the amount of CAT added, and were lowered by decreasing the amount of CAT to be added (e.g., from 10 to $2.5 \mathrm{mg}$ ), with exception of a few amino acids. Moreover, when the concentrations of amino acids were lowered to $2 \mathrm{~mm}$, the amino acids which developed color by Method I were only arginine and histidine.

of the amino acid related compounds tested, the color density of indole, which gives a deep color by the original procedure, was partially decreased.

Amine, and sugars and the related compounds (Table II). Among the compounds which give no color by the original procedure, only ethylenediamine and sucrose became to react with the phenol reagent. On the other hand, all the members which develop the color by the original procedure, with exception of tryptamine whose color became faint, produced a deeper color than that by the original procedure.

Nucleic acid bases and the related compounds (Table III). The compounds which give only slight or no color by the original procedure and produce the appreciable color by Method I were adenine, adenosine, and cytosine at $1 \mathrm{mg}$. On the other hand, it should be noted that

\section{Table I. Absorbances of Amino Acios and the Related Compounds in Method I}

Absorbances shown in parenthesis are those obtained by the original Lowry procedure. In this case, the absorbances below 0.01 are not shown in the table. Details of the method are described in the text.

\begin{tabular}{lcllcc}
\hline \multicolumn{1}{c}{ Compound } & $\mathrm{mM}$ & Absorbance & Compound & $\mathrm{mM}$ & Absorbance \\
\hline Alanine & 20 & 0.057 & Methionine & 20 & 0.578 \\
Arginine & 20 & $<1$ & Phenylalanine & 20 & 0.483 \\
Asparagine & 20 & $0.582(0.068)$ & Proline & 20 & 0.037 \\
Aspartic acid & 20 & 0.359 & Serine & 20 & 0.067 \\
Glycine & 20 & 0.042 & Threonine & 20 & 0.076 \\
Glutamic acid & 20 & 0.226 & Tryptophan & 1 & $0.308(0.790)$ \\
Glutamine & 20 & 0.065 & Tyrosine & 0.5 & $0.322(0.580)$ \\
Histidine & 20 & $>2(0.301)$ & Valine & 20 & 0.161 \\
Hydroxyproline & 20 & 0.026 & -Alanine & 20 & $<0.01$ \\
Isoleucine & 20 & 0.037 & Imidazole & 200 & $0.211(0.192)$ \\
Leucine & 20 & 0.053 & Indole & 1 & $0.223(0.560)$ \\
Lysine & 20 & 0.407 & & & \\
\hline
\end{tabular}


color yields of xanthine, uric acid, and guanine, which give the high absorbances by the original procedure, were markedly or partially decreased.

Phenols and the related compounds. Although all the tested compounds of this group give the blue color by the original procedure, the color densities of all of them were greatly or partially decreased (Table IV).

Miscellaneous compounds. As shown in Table V, a good many members of the compounds listed in the table produce the color by the original procedure. When Method I was applied to those members, the absorbance of penicillin $G$ showed a negligible value, and that of $\mathrm{CoCl}_{2}$ was partially decreased. On the contrary, the absorbances of aminomethylpropanediol, Tris, Tricine, TES, MOPS, CAPS, acetone, oxaloacetate, $\alpha$-ketoglutarate, and acetaldehyde were moderately or greatly increased.

Moreover, although data are not shown, the following compounds gave only the negligible absorbance: acetate, arsenite, borate, cacodylate, citrate, $\gamma$-collidine, fumarate, lactate, maleite, phosphate, succinate, tartrate, Vero-

Table II. Results with Amines, and Sugars and the Related Compounds Explanations for the table are the same as those in Table I.

\begin{tabular}{|c|c|c|c|c|c|}
\hline Compound & $\mathrm{mm}$ & Absorbance & Compound & $\%$ & Absorbance \\
\hline Benzylamine & 20 & 0.052 & Glucose & 5 & $>1(0.157)$ \\
\hline$n$-Buthylamine & 20 & $<0.01$ & Glucose-1-phosphate & 5 & 0.020 \\
\hline Ethylamine & 20 & 0.013 & Glucose-6-phosphate & 5 & $0.740(0.127)$ \\
\hline Etbylenediamine & 20 & 0.540 & Glucosamine & 0.4 & $0.163(0.073)$ \\
\hline Histamine & 20 & $>1(0.302)$ & Galactose & 5 & $0.950(0.332)$ \\
\hline Methylamine & 20 & $<0.01$ & Sucrose & 5 & $0.401(0.030)$ \\
\hline Tryptamine & 1 & $0.350(0.980)$ & Glycerol $^{a}$ & 23.4 & $0.366(0.168)$ \\
\hline
\end{tabular}

a The concentration was calculated from the specific gravity.

Table III. Results with Nucleic Acid Bases and the Related Compounds Explanations for the table are the same as those in Table $I$.

\begin{tabular}{lcclcc}
\multicolumn{1}{c}{ Compound } & $m g$ & Absorbance & Compound & mg & \multicolumn{1}{c}{ Absorbance } \\
\hline DNA & 1 & $0.065(0.067)$ & Uracil & 1 & 0.041 \\
RNA & 1 & $0.097(0.117)$ & Uridine & 1 & 0.049 \\
Adenine & 1 & $0.279(0.043)$ & Alloxan & 1 & $<0.01$ \\
Adenosine & 1 & $0.126(0.033)$ & Allantoin & 1 & $0.068(0.039)$ \\
Cytosine & 1 & 0.117 & Hypoxanthine & 1 & $0.135(0.155)$ \\
Cytidine & 1 & $0.037(0.037)$ & Xanthine & 0.1 & $0.184(>1)$ \\
Guanine & 0.05 & $0.228(0.685)$ & Uric acid & 0.1 & $0.025(0.980)$ \\
\hline
\end{tabular}

a In this case, the phenol reagent was added before the addition of the alkaline copper reagent. If both the reagents were added in the usual order, no color was developed with the indicated amount of uric acid.

Table IV. Results with Phenols and the Related Compounds Explanations for the table are the same as those in Table $\mathrm{I}$.

\begin{tabular}{|c|c|c|c|c|c|}
\hline Compound & $\mu \mathrm{g}$ & Absorbance & Compound & $\mu \mathrm{g}$ & Absorbance \\
\hline$m$-Cresol & 50 & $0.348(0.855)$ & Resorcinol & 50 & $0.013(0.930)$ \\
\hline Hydroquinone & 50 & $0.101(0.241)$ & Tannic acid ${ }^{a}$ & 50 & $0.028(0.612)$ \\
\hline Phenol & 25 & $0.358(0.559)$ & Thymol & 50 & $0.138(0.429)$ \\
\hline Pyrogallol & 50 & $0.017(0.237)$ & $\alpha-N a p h t h o l^{b}$ & 50 & $0.023(0.630)$ \\
\hline
\end{tabular}

a This hydrate water was unknown.

${ }_{b}$ Reaction between this compound and CAT was performed in $10 \%$ ethylalcohol $/ 2.5 \% \mathrm{TCA}$. 
Table V. Results with Miscellaneous Compounds Explanations for the table are the same as those in Table I.

\begin{tabular}{lrllll}
\multicolumn{1}{c}{ Compound } & $\mathrm{mM}$ & Absorbance & \multicolumn{1}{c}{ Compound } & $\mathrm{mM}$ & Absorbance \\
\hline $\begin{array}{l}\text { Aminomethyl- } \\
\quad \text { propanediol }\end{array}$ & 100 & $0.402(0.285)$ & Oxaloacetate & 20 & $0.648(0.100)$ \\
Tris & 200 & $0.785(0.157)$ & $\alpha$-Ketoglutarate & 20 & $0.352(0.188)$ \\
BES & 8 & $0.102(0.146)$ & Acetone & $20 \%$ & $0.434(0.052)$ \\
Bicine & 4 & $0.290(0.301)$ & Urea & 20 & 0.017 \\
CAPS & 200 & $0.318(0.143)$ & Acetaldehyde & $1.8 \%$ & $0.718(0.470)$ \\
HEPES & 4 & $0.225(0.232)$ & Formaldehyde & $18.6 \%$ & 0.023 \\
MES & 200 & $0.111(0.068)$ & $\left.\mathrm{NH}_{4}\right)_{2} \mathrm{SO}_{4}$ & $2 \%$ & 0.043 \\
MOPS & 80 & $0.330(0.136)$ & $\mathrm{CoCl}_{2}$ & 4 & $0.414(0.688)$ \\
PIPES & 40 & $0.218(0.235)$ & $\mathrm{MnCl}_{2}$ & 4 & $0.354(0.423)$ \\
TES & 200 & $0.730(0.043)$ & $\mathrm{NiCl}_{2}$ & 6 & $0.419(0.468)$ \\
Tricine & 200 & $0.350(0.067)$ & $\mathrm{Penicillin} \mathrm{G}$ & $250 \mu \mathrm{g}^{\mathrm{a}}$ & $<0.01$ \\
EDTA & 50 & $0.640(0.608)$ & & & $(0.335)$ \\
\hline
\end{tabular}

a This amount is equivalent to 396.3 units.

Table VI, Effects of Coexistence of Sulfhydryl Compounds in the Samples on ABSORBances OF SOME COMPOUNDS BY METHOD I

The following sulfhydryl compounds were used: $20 \mathrm{~mm}$ cysteine (CySH) and 2-mercaptoethanol (ME), and $10 \mathrm{~mm}$ dithiothreitol (DTT). Absorbances shown in parenthesis are those obtained by the original Lowry procedure. In this case, the absorbances below 0.01 are not shown in the table.

\begin{tabular}{|c|c|c|c|c|c|}
\hline \multirow{3}{*}{ Compound } & \multirow{3}{*}{ Concentration } & \multicolumn{4}{|c|}{ Absorbance } \\
\hline & & \multirow{2}{*}{$\begin{array}{l}\text { with only } \\
\text { CAT }\end{array}$} & \multicolumn{3}{|c|}{ with CAT and } \\
\hline & & & $\mathrm{CySH}$ & ME & DTT \\
\hline Arginine & $20 \mathrm{~mm}$ & $>1$ & 0.680 & $>1$ & $>1$ \\
\hline Histidine & 20 & $>2(0.301)$ & 0.820 & 0.740 & $>1$ \\
\hline Methionine & 20 & 0.578 & 0.228 & 0.230 & 0.355 \\
\hline Phenylalanine & 20 & 0.483 & 0.150 & 0.110 & 0.262 \\
\hline \multicolumn{6}{|l|}{ Aminomethyl- } \\
\hline propanediol & 100 & $0.402(0.285)$ & 0.342 & 0.378 & 0.285 \\
\hline Tris & 200 & $0.785(0.157)$ & 0.156 & 0.207 & 0.164 \\
\hline Tricine & 200 & $0.350(0.067)$ & 0.309 & 0.105 & 0.075 \\
\hline TES & 200 & $0.730(0.043)$ & 0.056 & 0.087 & 0.097 \\
\hline MOPS & 80 & $0.330(0.136)$ & 0.185 & 0.197 & 0.188 \\
\hline Sucrose & $5 \%$ & $0.401(0.030)$ & 0.265 & 0.381 & 0.398 \\
\hline Glycerol $^{a}$ & 23.4 & $0.366(0.168)$ & 0.143 & 0.146 & 0.112 \\
\hline
\end{tabular}

a The concentration was calculated from the specific gravity.

nal, $\mathrm{NaCl}, \mathrm{KCl}, \mathrm{CaCl}_{2}$, and $\mathrm{MgCl}_{2}$ at $200 \mathrm{~mm}$, ethyl-, methyl-, and isopropyl-alcohols in $20 \%$ solution and Triton $\mathrm{X}-100$, and sodium dodecylsulfate in $0.2 \%$ solution.

The absorbances of several compounds under the coexistence of SH compounds. As Method I was the procedure originally proposed for determining the protein content in the presence of $\mathrm{SH}$ compounds or some other reducing agents, ${ }^{2)}$ we examined effects of the coexistence of $\mathrm{SH}$ compounds in the sample on the absorbances obtained by Method I of the several compounds which gave the deep color by this method (see Tables $I \sim V$ ). As shown in Table VI, the absorbances increased by Method I of all tested compounds were greatly or partially decreased by the coexistence of one of SH compounds though the extent of decreases differed with the kind of SH compounds. It is 
especially noted that the absorbances of the tested compounds except several amino acids and sucrose were reduced to much the same values as those obtained by the original procedure.

\section{Results by Method II}

Histidine, histamine, and oxaloacetate gave very high absorbances at $20 \mathrm{~mm}$. The absorbances of arginine, asparagine, phenylalanine, glucose-6-phosphate, glucosamine, glycerol, TES, and acetaldehyde at the same concentrations as shown in Tables $I \sim \mathrm{V}$ showed only the slight increaments by this method. On the other hand, those of resorcinol, pyrogallol, and tannic acid were partially decreased. The absorbances of the other tested compounds were much the same of those obtained by the original procedure.

\section{DISCUSSION}

The Lowry procedure owes its wide use to its high sensitivity and simplicity. This method, however, has a gross drawback in that a host of compounds, including $\mathrm{SH}$ compounds, ${ }^{4)}$ phenols, ${ }^{1 /}$ several purine bases, ${ }^{1)}$ sucrose, ${ }^{5)}$ glycerol, ${ }^{6)}$ EDTA, ${ }^{7)}$ various components of buffers, ${ }^{8 \sim 10)}$ several metal ions, ${ }^{11\rangle}$ and penicillin derivatives ${ }^{12)}$ have been demonstrated to react with the Lowry reagent.

In the previous paper, we reported that Method I was an improved Lowry procedure for determining protein in the presence of $\mathrm{SH}$ compounds or some other reducing agents. ${ }^{23}$ The present results, however, show that many members of the tested compounds, especially at a high concentration, gave deeper color by Method I than by the original procedure (Tables I V). Although the detailed mechanisms of the increases in reactivities of these compounds by Method I are unknown at present, it seems to be indisputable that those increases result from the second reactions between the Lowry reagent the various intermediate products produced by the oxidation with CAT of those compounds under the acid condition.
In the case of amino acids, it is known that all amino acids can reduce the phenol reagent after being treated with $\mathrm{Co}(\mathrm{II})^{13)}$ though none of those, except histidine, tryptophan, and tyrosine, gives the blue color by the Lowry procedure. ${ }^{14)}$ In addition, it is known that the several amino acids react with CAT to yield $\mathrm{CO}_{2}$ and the corresponding aldehydes or nitrils, and that CAT itself simultaneously converts to $p$-toluenesulphonamide or benzaldehyde- $p$-sulphonamide. ${ }^{15,16)}$ It is speculated from these facts that the increases of absorbances of amino acids by Method I may be due to the stable chelate formation between copper and the secondary materials produced from amino acids by CAT treatment. The absorbances of amino acids by Method I, thereupon, were compared with those of amino acids by using the copper-free alkaline reagent. No definite effect of copper in Method I, however, was obtained in the many amino acids except the several (data not shown).

Apart from amino acids, among the tested compounds almost all members of the compounds whose absorbances were increased by Method I possessed the plural hydroxy methyl or amino methyl groups, or vicinal glycolic groups, and/or a carbonyl group in their molecules, though the absorbance of glucose-1phosphate, which contained vicinal glycolic groups, but no carbonyl group, was not increased. This fact suggests an important contribution of those groups to the increases of absorbances by Method I. Although the detailed mechanisms of this contribution are unknown, it is possible that by CAT treatment in the acid medium these groups are converted to the other groups which react with the phenol reagent in the Lowry procedure. For example, it was found that although the absorbance of Tris by the original procedure was independent of the concentration as shown by $\mathrm{Ji}^{10}{ }^{10}$ that of Tris by Method I became to depend on it. Moreover, when Tris of $100 \mathrm{~mm}$ concentration was used, its color by the original procedure disappeared almost completely by using the copper-free alkaline reagent, but by Method I the color was only faded about $30 \%$ by use of 
the same reagent.

Among the compounds whose absorbances were increased by Method I, the absorbances of the several ones such as Tris, Tricine, sucrose and glycerol, which are widely used as buffer substance and as media for the cell fractionation or the stabilization of enzyme protein, were markedly decreased when Method I was used in the presence of one of $\mathrm{SH}$ compounds (Table VI). These results show that the protein assay in the presence of indicated concentration of them is permitted by Method I, if one of SH compounds coexists in the sample and if a standard curve for protein samples containing corresponding amount of these compounds is prepared.

On the other hand, Method I greatly or partially decreased the absorbances of some compounds such as phenols or several purine bases. These results suggest that the protein determination in the presence of these compounds is possible by Method I. This will be reported elsewhere.

When an attention is turned to Method II, the number of the compounds whose absorbances were increased by this method was only a few. Among these compounds, although the absorbances of histidine, histamine, and oxaloacetate at $20 \mathrm{~mm}$ were too high to permit the determination of protein, it was possible to determine the protein content in the presence of other compounds by using the corresponding blank and the protein standard curve under the same condition as the samples.

\section{REFERENCES}

1) O. H. Lowry, N. J. Rosebrough, A. L. Farr and R. J. Randall, J. Biol. Chem., 193, 265 (1951).

2) M. Higuchi and F. Yoshida, Anal. Biochem., 77, 542 (1977).

3) M. Higuchi and F. Yoshida, Agric. Biol. Chem., 42, 75 (1978).

4) C. G. Vallejo and R. Lagunas, Anal. Biochem., 36, 207 (1970).

5) B. Gerhardt and H. Beevers, ibid., 24, 337 (1968).

6) M. K. Zishkaand and J. S. Nishimura, ibid., 34, 29 (1970).

7) A. R. Neurath, Experientia, 22, 290 (1966).

8) R. Rej and A. H. Richard, Anal. Biochem., 62, 240 (1974).

9) J. D. Gregory and S. W. Sajdera, Science, 169, 97 (1970).

10) T. H. Ji, Anal. Biochem., 52, 517 (1973).

11) M. Higuchi and F. Yoshida, ibid., 65, 591 (1975).

12) D. J. Silverman, ibid., 27, 189 (1969).

13) S. Matsushita and N. Iwami, Agric. Biol. Chem., 30, 1061 (1966); S. Matsushita, N. Iwami and Y. Nitta, Anal. Biochem., 16, 365 (1966).

14) S. Chou and A. Goldstein, Biochem. J., 75, 109 (1960).

15) H. D. Dankin, ibid., 11, 79 (1917).

16) D.S. Mahadevappa and H.M. K. Naidu, Curr. Sci., 45, 652 (1976). 